\title{
Simulação baseada em System Dynamics para avaliação de cenários sobre geração e disposição de resíduos sólidos urbanos
}

\author{
Eugênio de Oliveira Simonetto ${ }^{\mathrm{a} *}$, Mauri Leodir Löbler $^{\mathrm{b}}$ \\ a*eosimonetto@ufsm.br, UFSM, Brasil \\ blobler@ccsh.ufsm.br, UFSM, Brasil
}

\begin{abstract}
Resumo
0 artigo apresenta o desenvolvimento, validação e experimentação de um modelo de simulação utilizando a metodologia System Dynamics, a qual permite avaliar e analisar cenários acerca da geração e disposição final dos resíduos sólidos urbanos (RSU). Para tanto, levou-se em consideração: a taxa de crescimento vegetativo populacional (nascimentos e mortes), percentual de resíduo sólido urbano enviado para cada tipo de destino final e a quantidade de resíduos gerada por habitante. A validação do modelo foi através da análise de cenários futuros para um determinado município da região sul do Brasil. Para a modelagem e execução do sistema foi utilizado o software Vensim, da Ventana Systems. Com os resultados gerados pelo modelo de simulação, os gestores da área podem, antecipadamente, discutir, avaliar e decidir possíveis medidas necessárias para melhorias ou adaptações na gestão de resíduos sólidos urbanos.
\end{abstract}

Palavras-chave

Simulação computacional. System Dynamics. Gestão de resíduos sólidos urbanos. Processo decisório. Resíduos sólidos.

\section{Introdução}

A preocupação da comunidade com os limites do desenvolvimento do planeta teve início na década de 1960, quando iniciaram-se as discussões sobre os riscos da degradação do meio ambiente. Tais discussões levaram os pesquisadores e a comunidade a concluir que, mantido o ritmo de industrialização, poluição e exploração de recursos naturais, o limite de desenvolvimento do planeta seria atingido dentro de cem anos, no máximo (MEADOWS; MEADOWS, 1972; MEADOWS; RANDERS; MEADOWS, 2004).

0 manejo ambientalmente correto dos resíduos sólidos encontra-se dentre as questões mais importantes para a manutenção da qualidade do meio ambiente e, principalmente, para que se alcance um desenvolvimento sustentável e ambientalmente correto em todos os países (ZUTSHI; SOHAL, 2002). Apesar de toda a preocupação da comunidade internacional com a qualidade do meio ambiente, o que acontece na maioria das vezes é um desenvolvimento não sustentável na gestão dos resíduos sólidos municipais, pois os métodos para a coleta, transporte e depósito dos resíduos sólidos não levam em conta as consequências que o mau uso e o tratamento inadequado deles podem acarretar no meio ambiente em um futuro bem próximo.

Para a compreensão do trabalho, são considerados resíduos sólidos todos os restos domésticos e resíduos não perigosos, tais como resíduos comerciais, institucionais, de podas e de construções. Em alguns países, o sistema de gestão dos resíduos sólidos também se ocupa dos resíduos humanos, tais como excrementos, cinzas de incineradores e de instalações de tratamento de esgoto (BARLAZ et al., 1995).

A gestão de resíduos sólidos é um tópico que vem atraindo a atenção de pesquisadores em pesquisa operacional (PO) e modelagem de sistemas nos últimos anos (BAN1 et al., 2009). Vários autores (BHAT, 1996; HUANG; BAETZ; PATRY, 1998; CHANG; WEI, 2000; CHANG; PARVATHINATHAN; BREEDEN, 2008; COSTI et al., 2004; SIMONETTO; BORENSTEIN, 2007; 
SUFIAN; BALA, 2007; TANSKANEN, 2000; TUNG; PINNOI, 2000; WEINTRAUB; MARTELL; GUNN, 1998) já utilizaram técnicas e métodos da PO para desenvolver estudos nessa área específica, embora esses estudos sejam bastante escassos no Brasil. A utilização de técnicas quantitativas na gestão de resíduos sólidos surge como uma alternativa viável para o tratamento da complexidade inerente ao processo, pois através do uso dessas ferramentas pode-se representar uma situação do mundo real, estudar seu comportamento e tomar decisões com base nas conclusões extraídas.

0 objetivo principal do artigo é apresentar o desenvolvimento e validação de um modelo de simulação computacional que permita aos gestores da área de resíduos sólidos urbanos (RSU) avaliarem e analisarem cenários acerca da geração e destinação final dos RSU. Para tal, o problema de pesquisa consistiu na investigação, definição e validação das variáveis componentes do modelo de simulação, bem como da concepção e validação dele. Para o desenvolvimento do modelo computacional foram utilizadas técnicas oriundas da área de System Dynamics (DAELLENBACH; McNICKLE, 2005; GHARAJEDAGHI, 2006). 0 uso dessas técnicas objetiva agregar qualidade ao processo decisório, pois muitas decisões sobre o planejamento da gestão dos resíduos sólidos são tomadas baseadas somente na experiência dos gestores (CHANG; WEl, 2000). Tal fato, segundo esses autores, contribui para o alto custo e o baixo desempenho da gestão de resíduos nos municípios. Para a validação do modelo foram analisados possíveis cenários futuros, porém utilizando-se dados reais acerca da geração e da disposição final dos RSU de um determinado município.

0 trabalho está organizado da seguinte forma: na seção 2 é apresentada a metodologia de pesquisa utilizada para o desenvolvimento do estudo. $\mathrm{Na}$ seção 3 é apresentada uma introdução à simulação computacional e à metodologia System Dynamics, na seção 4 são apresentados conceitos sobre resíduos sólidos, tratamentos e disposição final. Na seção 5 são descritos o problema de modelagem, as variáveis componentes e o modelo desenvolvido. Na seção 6 são apresentados a validação, os cenários de simulação e o experimento utilizando o modelo, bem como a discussão de resultados. As considerações finais são apresentadas na seção 7 .

\section{Metodologia da pesquisa}

Simon (1990) afirma que a modelagem de sistemas pode ser classificada em dois tipos, predição e prescrição. Embora o fascínio por predizer o futuro, que é natural no ser humano, afinal é o futuro dele mesmo, uma modelagem que assuma a predição do comportamento temporal de grandes sistemas não tem chances de sucesso absoluto. A modelagem com maiores probabilidades de sucesso é a prescritiva. Segundo Simon (1990), tomar o problema levantado pelo Clube de Roma, da predição do "inverno nuclear", e abordá-lo como um problema prescritivo e não como um problema preditivo seria o caminho mais indicado, pois as questões importantes a serem respondidas surgiriam. Qual é a população que pode ser mantida na terra em "estado estacionário" com um padrão de vida razoável, e qual é o estado estacionário de quantidade de energia produzida possível de ser mantido sem que danos sérios sejam infringidos ao meio ambiente?

Nesse trabalho, a metodologia de pesquisa adotada para o desenvolvimento do modelo computacional foi baseada na metodologia proposta por Law e Kelton (1991) e consistiu das seguintes etapas: (1) estudos exploratórios em artigos científicos, manuais de referência e entrevistas com gestores da área de resíduos sólidos, nas quais o problema foi caracterizado e estruturado; (2) desenvolvimento da solução, pela construção de modelos formais capazes de representar o problema; (3) implementação computacional da solução, utilizando-se o simulador Vensim (VENTANA SYSTEMS, 2011) da área de System Dynamics; (4) validação da solução, através de testes em laboratório e em campo, para verificar se os resultados obtidos estão de acordo com a realidade observada, bem como através da simulação de um experimento para tal, utilizando-se três cenários.

Os cenários que foram utilizados para a validação do modelo foram gerados a partir de análises em que foram utilizados dados históricos relativos aos censos populacionais de 2000 e 2010 (INSTITUTO..., 2010), o Panorama dos Resíduos Sólidos no Brasil - 2010 (ASSOCIAÇÃO..., 2011), um boletim informativo sobre tipos de disposição final na Comunidade Europeia (EUROPEAN..., 2011), bem como o Diagnóstico de Manejo de Resíduos Sólidos (BRASIL, 2010) e também contou com a participação de pesquisadores e especialistas na área.

\subsection{Formulação de hipótese dinâmica}

Segundo Silva (2006), a hipótese dinâmica visa trabalhar a teoria do problema, analisando o seu comportamento e observando quais variáveis fazem parte do sistema. Neste artigo incorporaram-se três variáveis básicas: variação populacional, geração de resíduos e destino final dos resíduos (subdividido nos possíveis destinos finais). A primeira delas (variação populacional) entendeu-se como a de maior dificuldade de controle, visto que o local utilizado já cresce a 
uma taxa diminuta de 0,7\% ao ano, manteve-se essa taxa de crescimento fixa nos três cenários.

A geração de resíduos atual é de $1,213 \mathrm{~kg} /$ pessoa, utilizando a Holanda, que é padrão mundial em tratamento de RSU, o nível de produção chega a $1,466 \mathrm{~kg} /$ pessoa. 0 modelo proposto utilizou essas variações na modelagem. Quanto ao destino final dos resíduos, utilizou-se variação nas taxas destinadas ao aterro sanitário, à reciclagem, à compostagem, à incineração e ao tratamento inadequado. Tal variação teve por embasamento a dinâmica das taxas médias nacionais e, também, taxas de tratamento de RSU consideradas adequadas, no caso, taxas holandesas.

0 objetivo dessa etapa é formular uma hipótese que explique a dinâmica como consequência da estrutura interna do sistema por meio da interação entre as variáveis e os agentes representados no modelo, incluindo regras de decisão (STRAUSS, 2010). Assim, a hipótese dinâmica do modelo de System Dynamics deste trabalho é definida a seguir:

A variação populacional associada à quantidade de resíduos gerada pela população possui influência direta na quantidade total de resíduos sólidos urbanos gerada, bem como na quantidade de RSU destinada ao aterro sanitário, à reciclagem, ao destino final inadequado e, também, na área total para disposição final dos mesmos em aterro sanitário.

\section{Simulação computacional}

A simulação computacional de sistemas consiste na utilização de um conjunto de métodos e técnicas matemáticas, com o objetivo de imitar o comportamento de sistemas reais, geralmente utilizando-se de computadores e softwares (KELTON; SADOWSK1; SADOWSK1, 1998). Ela pode ser definida como um processo de projetar um modelo de um sistema real e procedimentos de experimentos com esse modelo, com o propósito de verificar o comportamento do sistema, ou até mesmo avaliar estratégias para a sua operação (PEGDEN; SHANON; SADOWSK1, 1995).

Através do uso da simulação pode-se imitar o comportamento de, praticamente, qualquer tipo de operação ou processo do mundo real (LAW; KELTON, 1991). Uma das principais etapas de um estudo de simulação consiste na criação de um modelo lógico. Assim, um modelo lógico consiste em um conjunto de suposições e aproximações, devidamente quantificadas e estruturadas, que visam representar o comportamento do sistema real sob determinadas condições, utilizando-o para predizer e comparar alternativas lógicas passíveis de serem simuladas.

Um modelo é utilizado também quando se deseja aprender alguma coisa sobre o sistema real que não se pode observar ou experimentar diretamente, ou pela inexistência do sistema real, ou pela dificuldade de sua manipulação, ou pela impossibilidade de sujeitá-lo ao ensaio sem incorrer em custos elevados e gastos de tempo. A quantidade de simplificações impostas ao modelo influenciará em razão direta na distorção entre os resultados obtidos pelo modelo e pelo sistema real. Portanto, esse método é uma das ferramentas de análise mais poderosas disponíveis para a representação de sistemas complexos (KELTON; SADOWSKI; SADOWSK1, 1998).

A simulação possui várias aplicações no cotidiano, nas áreas mais diversas, que vão desde o controle de produção na área de alimentos (PIDD, 1987), do controle do fluxo de papéis em um escritório (DAVIES, 1994), do controle do trânsito nas cidades (SALT, 1991) até o controle do transporte de instrumentos cirúrgicos dentro de um hospital (CERIC, 1990). Atualmente, a simulação computacional também vem sendo utilizada como auxiliar em problemas relacionados à gestão ambiental, tais como gestão de recursos hídricos (WINZ; BRIERLEY, 2007), gestão de resíduos sólidos (SUFIAN; BALA, 2007), análise de mudanças climáticas (ZANIS et al., 2011) e ao estudo do comportamento de nano elementos (NANOHUB, 2012).

Uma das técnicas utilizadas para a simulação computacional é a metodologia de System Dynamics, a qual é apresentada a seguir na subseção 3.1.

\subsection{System Dynamics}

A metodologia System Dynamics (SD) foi desenvolvida durante a década de 1950 pelo engenheiro Jay Forrester, do Instituto Tecnológico de Massachussets (MIT). Sua primeira aplicação foi numa análise de uma empresa americana, mais precisamente o estudo das oscilações nas vendas dessa empresa. Esse estudo foi publicado com o nome Industrial Dynamics.

Em 1969 foi publicado o livro Dinâmica urbana, no qual é apresentado o modelo de System Dynamics aplicado na modelagem de cidades. Esse trabalho, posteriormente, viria a servir de base para que Meadows e Meadows (1972) desenvolvessem o trabalho Os limites do crescimento, apresentado ao Clube de Roma no início da década de 1970. Esses trabalhos e suas repercussões popularizaram o System Dynamics no mundo inteiro.

A metodologia System Dynamics permite o estudo do comportamento dos sistemas ao longo do tempo de maneira a possibilitar a avaliação das consequências de nossas decisões (DAELLENBACH; McNICKLE, 2005). Por tal motivo e a necessidade de estudar o 
comportamento da geração e disposição final dos resíduos em um horizonte temporal futuro, decidiu-se utilizá-la na modelagem e simulação computacional.

Um modelo de SD pode ser interpretado como a estrutura resultante da interação de políticas. Essa estrutura é formada por dois componentes principais, que são os estoques e os fluxos. Os estoques retratam as variáveis que são acumuladas e os fluxos são as funções de decisão ou políticas de um sistema. Esses componentes podem estar organizados na forma de relações circulares de causa e efeito, conhecidas como feedback de balanço ou de reforço, e estão sujeitos a defasagens de tempo no sistema. Sufian e Bala (2007) utilizaram essa abordagem para a modelagem do sistema de gestão de resíduos sólidos da cidade de Dhaka-Bangladesh. Outros autores que também utilizaram tal metodologia foram Abeliotis et al. (2009), Dyson e Chang (2005) e Kum, Sharp e Harnpornchai (2005).

\subsubsection{Componentes do modelo}

Em System Dynamics, um modelo é construído com basicamente quatro componentes: estoques, fluxos, auxiliares e conectores. Os estoques são variáveis de estado e podem ser considerados como repositórios onde algo é acumulado, armazenado e potencialmente passado para outros elementos do sistema (DEATON; WINEBRAKE, 2000). Eles fornecem uma visão de como está o sistema em qualquer instante do tempo. E quaisquer mudanças nos estoques, que ocorrem devido à ação dos fluxos, demandam certo tempo, ou seja, não são instantâneas (COVER, 1996).

Os fluxos, por sua vez, são variáveis de ação e podem alterar os estoques, aumentando ou diminuindo seu volume. Os auxiliares servem para formular os dados para definir as equações dos fluxos. Eles servem para combinar, através de operações algébricas, fluxos, estoques e outros auxiliares.

Os auxiliares são usados para modelar as informações e não o fluxo físico, sendo capaz de se alterarem instantaneamente, sem atrasos (COVER, 1996). Os conectores representam as interrelações entre todos os componentes do sistema. São essas inter-relações que ligam os componentes que formarão a expressão matemática (DEATON; WINEBRAKE, 2000). A Figura 1 demonstra cada um dos componentes de um modelo de System Dynamics.

\section{Resíduos sólidos}

0 descarte dos resíduos é uma operação que envolve as fases de limpeza, coleta e destinação final. Esses serviços são considerados tarefas tipicamente municipais e, por isso, são organizados pelos governos

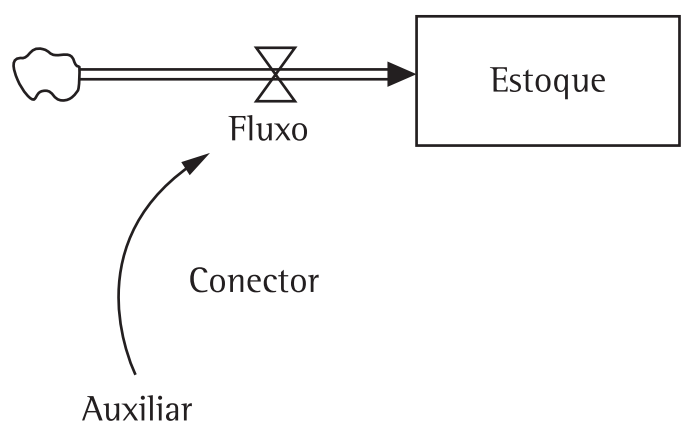

Figura 1. Componentes de um modelo de System Dynamics.

locais. Entre eles, a destinação final do lixo é uma preocupação que vem ganhando relevância, devido às suas implicações com a qualidade de vida das populações e a necessidade de aprimorar as técnicas de manejo dos resíduos sólidos urbanos (MONTEIRO et al., 2001).

0 manejo ambientalmente correto dos resíduos deve ir além do simples depósito ou aproveitamento por métodos seguros, deve-se buscar no conceito de desenvolvimento sustentável a forma de tratamento de resíduos sólidos, ou seja, a mudança dos padrões de produção e consumo. A utilização deste conceito (desenvolvimento sustentável) é uma forma de conciliar o desenvolvimento com o bem-estar do meio ambiente (RESOL..., 2008).

A estrutura da ação necessária deve apoiar-se em uma hierarquia de objetivos e centrar-se nas quatro principais áreas de programas relacionadas com os resíduos, a saber: (RESOL..., 2008): (a) redução dos resíduos ao mínimo; (b) aumento ao máximo da reutilização e reciclagem ambientalmente saudáveis dos resíduos; (c) promoção do depósito e tratamento ambientalmente saudáveis dos resíduos; e (d) ampliação do alcance dos serviços que se ocupam dos resíduos.

Essas áreas de programas são relacionadas entre si e atuam integradas para constituir uma estrutura ambientalmente saudável para o manejo dos resíduos sólidos. No entanto, é preciso uma estrutura bem definida e projetada para o gerenciamento sustentável dos resíduos sólidos.

A coleta do lixo é o segmento que mais se desenvolveu dentro do sistema de limpeza urbana e o que apresenta maior abrangência de atendimento junto à população, ao mesmo tempo em que é a atividade do sistema que demanda maior percentual de recursos por parte da municipalidade (MONTEIRO et al., 2001; O'LEARY et al., 1999; HUANG; BAETZ; PATRY, 1998). Esse fato se deve à pressão exercida pela população e pelo comércio para que se execute a coleta com regularidade, evitando-se assim o incômodo da 
convivência com o lixo nas ruas. Contudo, essa pressão tem geralmente um efeito seletivo, ou seja, quando a administração municipal não tem meios de oferecer o serviço a toda a população, prioriza os setores comerciais, as unidades de saúde e o atendimento à população de renda mais alta. Um dos grandes problemas enfrentados pelas administrações municipais para a coleta nas áreas mais carentes dos municípios é a ausência de infraestrutura viária adequada, o que exige a adoção de sistemas alternativos que apresentam baixa eficiência e, portanto, custo mais elevado. A disposição final dos resíduos é um dos problemas mais críticos enfrentados pelas municipalidades.

Analisando-se apenas os resíduos sólidos urbanos percebe-se claramente que a ação generalizada das administrações públicas ao longo dos anos foi apenas afastar das zonas urbanas o resíduo sólido coletado, depositando-o, muitas vezes, em locais absolutamente inadequados, como encostas e rios, nos populares "lixões". Outra forma de disposição final dos resíduos sólidos são os aterros sanitários que, por sua vez, são projetados de forma a minimizar os impactos à população e ao meio ambiente.

Com relação ao tratamento dos resíduos sólidos há unidades de compostagem e reciclagem instaladas no Brasil, que utilizam tecnologia simplificada, com segregação manual, no caso dos recicláveis, e compostagem a céu aberto, com posterior peneiramento. As poucas usinas de incineração existentes, utilizadas exclusivamente para incineração de resíduos de serviços de saúde e de aeroportos, em geral não atendem aos requisitos mínimos ambientais da legislação brasileira (MONTEIRO et al., 2001). Ainda, segundo Monteiro et al. (2001), outras unidades de tratamento térmico desses resíduos, tais como autoclavagem, microondas e outros vêm sendo instaladas mais frequentemente em algumas cidades brasileiras, mas os custos de investimento e operacionais ainda são muito altos.

\section{Variáveis e o modelo de simulação}

Com o passar dos anos, as áreas de destinação final dos resíduos sólidos urbanos podem se tornar escassas, obrigando os responsáveis pela sua gestão a agirem, de forma antecipada, na prevenção de problemas que poderão advir de tal escassez (CHANG; WEl, 2000; MONTEIRO et al., 2001). A concepção do modelo desenvolvido neste trabalho deu-se após a análise do trabalho desenvolvido por Chang e Wei (2000) em Taohsiung, Taiwan, o qual descreve a rapidez com que se esgotam os espaços físicos de armazenamento dos resíduos sólidos e, também, pelo tempo consumido no processo de determinação da área para novos aterros e construção de novos incineradores. Em Taohsiung, a alternativa foi a busca pelo aumento das taxas de reciclagem de resíduos.

Além do problema recorrente relativo à falta de espaço para disposição final dos RSU, outro fator que corrobora para a análise de possibilidades futuras com relação a esses resíduos é o aumento significativo das taxas de sua geração, associado ao aumento populacional. 0 crescimento da quantidade gerada de resíduos em grande parte tem por disposição final os aterros e até mesmo tratamento inadequado ("lixões" e aterros controlados). Além disso, as taxas de reaproveitamento, ou por reciclagem ou por compostagem, continuam baixas como há dez anos (NETTO, 2001).

0 modelo desenvolvido foi concebido com o intuito de auxiliar os responsáveis pela gestão de RSU no processo decisório no que se refere ao planejamento para um melhor aproveitamento e destinação dos resíduos gerados pela população, um melhor dimensionamento de áreas para o depósito dos resíduos sólidos urbanos e, por fim, permitir que se avaliem, antecipadamente, políticas para a gestão integrada de resíduos sólidos, de forma a minimizar os impactos ambientais provocados pela crescente geração desses resíduos, associada ao crescimento populacional.

Tais decisões poderão envolver a construção de novos aterros sanitários, a busca da elevação da taxa de reciclagem e compostagem, através de campanhas de conscientização da população, bem como outras análises e observações de interesse dos gestores, desde que sejam passíveis de execução no modelo de simulação desenvolvido. Cabe ressaltar que o modelo foi construído de modo a simplificar a interação usuário-máquina, para que as análises do tipo "o que se" (what-if), comuns em modelos de simulação, sejam de fácil e simples execução. No modelo de simulação, as análises what-if são de uso simples, pois quando da modificação nos valores das taxas e variáveis, novos resultados são gerados, para posterior análise por parte dos gestores. Uma das facilidades desse tipo de análise é a geração de gráficos comparativos com os resultados dos vários cenários simulados.

Para a identificação e definição das variáveis componentes do modelo de simulação (Figura 2) foram utilizados trabalhos acadêmicos e profissionais na área da gestão de resíduos sólidos (CHANG; WEl, 2000; O'LEARY et al., 1999; MONTEIRO et al., 2001; UNITED..., 2005; EUROPEAN..., 2011, BRASIL, 2010). A validação dessas foi executada com a participação de gestores da área. As variáveis selecionadas, bem como suas inter-relações com outras variáveis, as quais influenciam nos valores totais de geração e disposição final dos resíduos sólidos urbanos, são: 


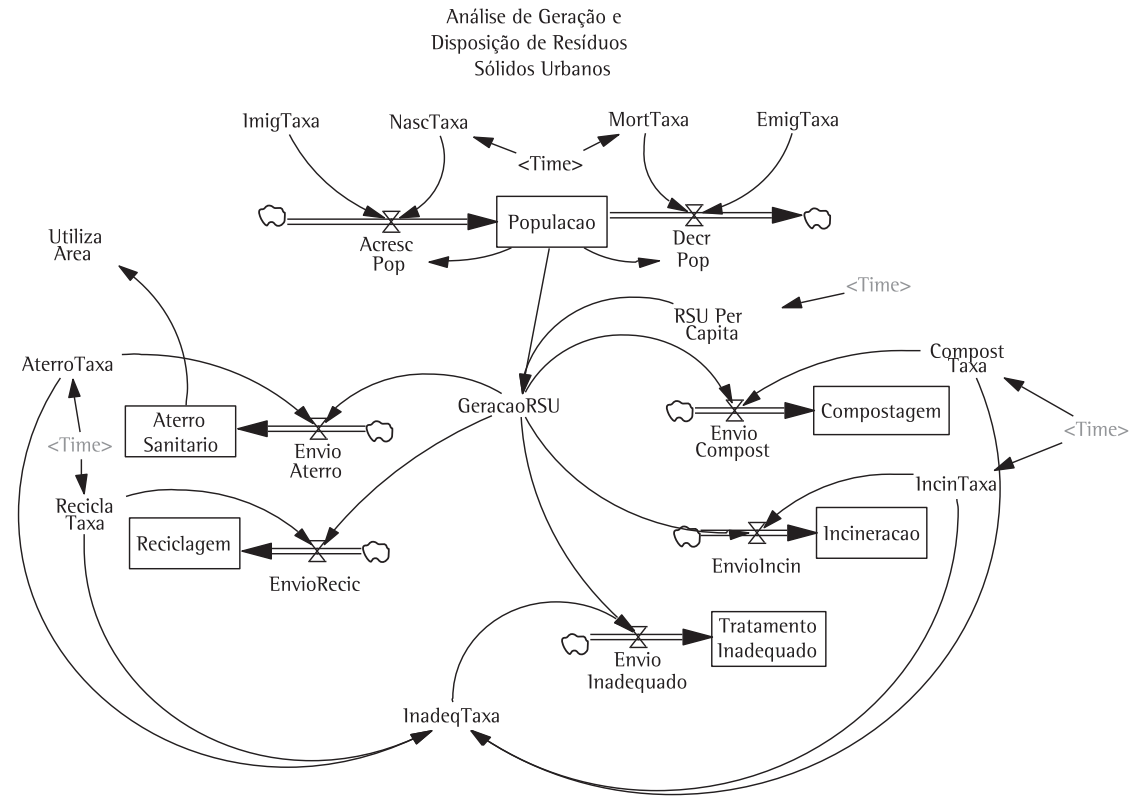

Figura 2. Modelo de simulação desenvolvido.

- A taxa de nascimento anual (NascTaxa), a taxa de mortalidade anual (MortTaxa), a taxa de imigração anual (ImigTaxa) e a taxa de emigração anual (EmigTaxa), todas essas influenciando diretamente os fluxos de entrada e saida populacional (AcrescPop e DecrPop), os quais determinam a população total (Populacao) do município. Ou seja, foi utilizada no modelo a taxa de crescimento natural (total de nascimentos - total de mortes), a qual corresponde à única forma possível de crescimento ou redução da população mundial e que, quando se analisa o crescimento de áreas específicas; devem ser consideradas, também, as migrações. Essas variáveis são representadas pelas Equações 1, 2 e 3 na formulação matemática do modelo de equações diferenciais ordinárias (Figura 3);

- a quantidade média de resíduos (RSUPerCapita) gerada por cada habitante multiplicada pela população total do município resulta na quantidade total de resíduos (GeracaoRSU) do município. As variáveis descritas são representadas na Equação 4 do modelo matemático da Figura 3;

- a taxa percentual absorvida por cada destino final dos resíduos (AterroTaxa, ReciclaTaxa, CompostTaxa, IncinTaxa, InadeqTaxa) , quais sejam, aterro sanitário, reciclagem, compostagem, incineração e, caso ocorra, o percentual enviado a formas de tratamento inadequadas (a taxa InadeqTaxa é dependente do valor de todas outras taxas, pois só ocorrerá a sua utilização se as outras formas de tratamento não conseguirem encarregar-se da quantidade total de RSU gerados). As variáveis

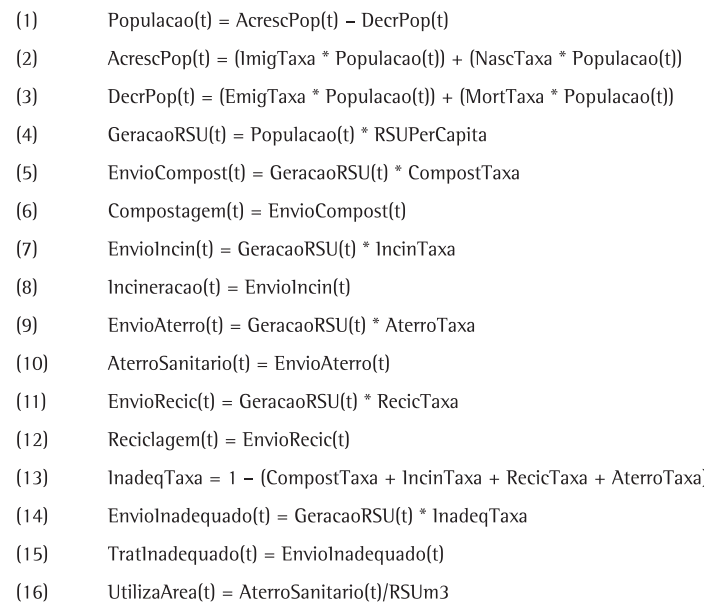

Figura 3. Formulação matemática do modelo de simulação desenvolvido.

descritas são utilizadas nas Equações 5, 7, 9, 11, 13 e 14, as quais podem ser visualizadas na Figura 3;

- os fluxos de entrada de resíduos nos diferentes tipos de disposição final (EnvioAterro, EnvioRecic, Enviolncin, Enviolnadequado, EnvioCompost), os quais representam a totalidade anual de resíduos sólidos destinada a cada uma das disposições finais, são obtidos através do produto da quantidade total de resíduos pela taxa percentual de cada destino final. As equações (ver Figura 3) que representam as variáveis relativas aos fluxos de entrada estão descritas no modelo matemático: são as Equações 5 , $7,9,11$ e 14 
- a quantidade total acumulada enviada para cada tipo de destino final é representada pelas variáveis de nível AterroSanitario (aterro sanitário), Reciclagem (reciclagem), Incineracao (incineração), Compostagem (compostagem) e Tratamentolnadequado (tratamento inadequado). As variáveis relativas aos possíveis destinos finais dos RSU estão descritas nas equações (6), (8), (10), (12) e (15), na Figura 3;

- por fim, a área total ocupada pelos resíduos sólidos é determinada pela variável UtilizaArea, a qual é obtida através da divisão da quantidade total de resíduos enviada ao aterro sanitário pela quantidade de resíduo sólido que ocupa $1 \mathrm{~m}^{3}$. A equação (16) na Figura 3 que representa o cálculo da área total utilizada pelo aterro.

Na Figura 4 é apresentada uma estrutura de fluxograma da simulação (algoritmo), de modo a facilitar o entendimento do modelo de simulação pelos usuários.

\section{Validação e experimentação do modelo}

A validação de um modelo de apoio ao processo decisório, segundo Finlay (1994, p. 40), é “o processo de teste de conformidade entre o comportamento do modelo e o sistema do mundo real que está sendo modelado". Finlay (1994) também afirma que não é possível um modelo representar o sistema do mundo real na sua totalidade, porém afirma existir a possibilidade de definirem-se relacionamentos entre os componentes do modelo, os quais permitam uma representação aceitável do mundo real.

Para Borenstein e Becker (2000), a validação consiste em testar as concordâncias entre o
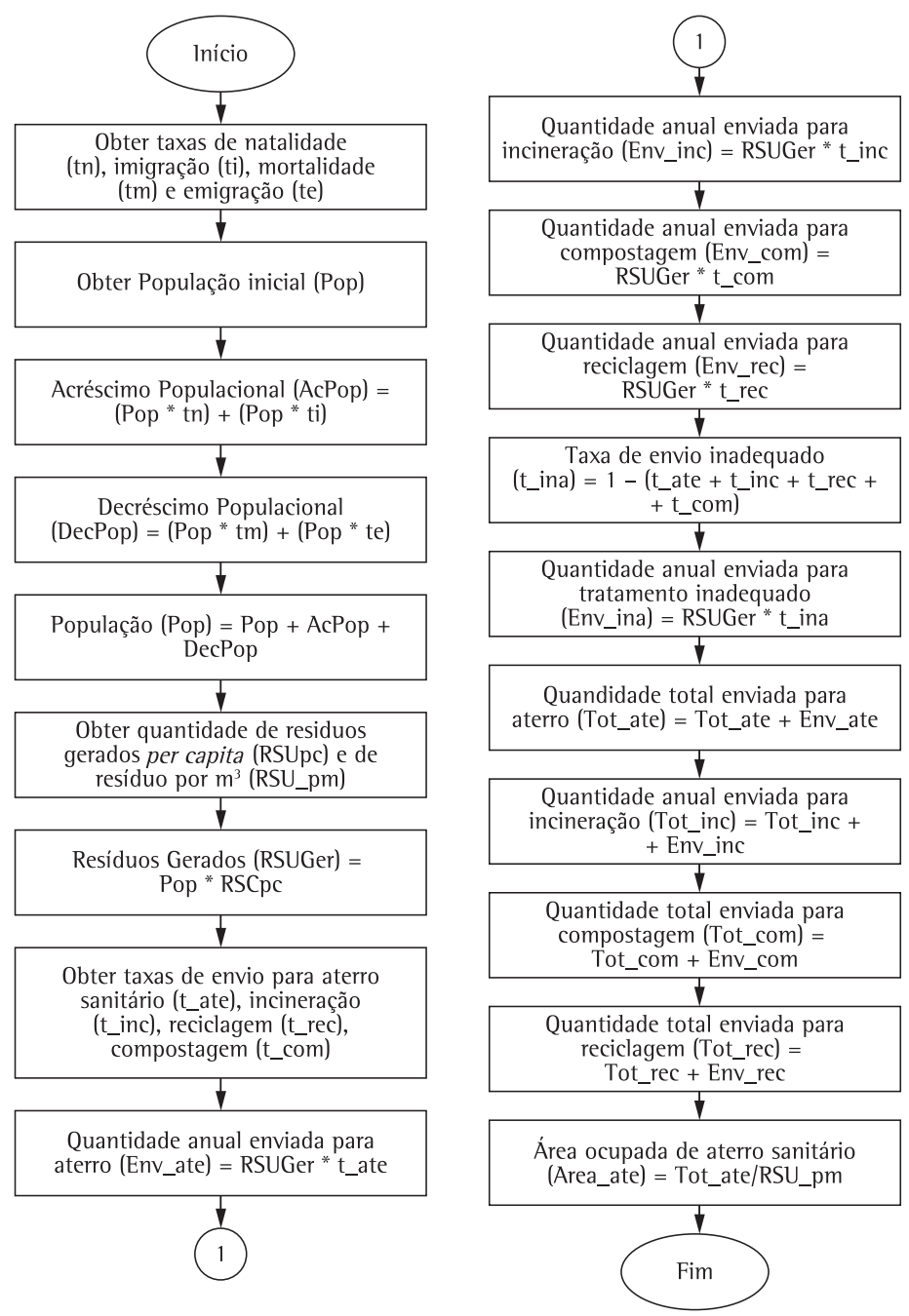

Figura 4. Fluxograma de uso do modelo de simulação. 
comportamento do sistema e o do mundo real sendo modelado. 0 trabalho dos autores refere-se à validação de sistemas. Os mesmos autores a dividem em duas principais categorias: a validação quantitativa e a qualitativa. As técnicas qualitativas dependem da avaliação subjetiva do sujeito. A validação quantitativa emprega a estatística para verificar o desempenho do sistema em campo de testes. Os tipos de validação podem ser visualizados a seguir na Tabela 1 .

A validação do modelo de simulação deu-se em diferentes etapas do seu desenvolvimento. Na primeira fase de validação (modelo conceitual) foram utilizados dados de artigos científicos, de manuais técnicos referentes à área de gestão integrada de resíduos sólidos e, também, obtidos com a participação de especialistas na área de gestão de resíduos, aqui fica caracterizada a validação nominal, visto que foram utilizados especialistas para definirem as variáveis importantes na modelagem proposta. Na segunda fase de validação, quando da implementação no simulador Vensim (VENTANA SYSTEMS, 2011), foram utilizados dados históricos sobre população e gestão de RSU (INSTITUTO..., 2002) para a verificação da integração entre os módulos componentes do modelo, bem como dos resultados gerados, pois foram avaliadas as saídas produzidas pelo modelo de simulação a partir de dados reais fornecidos - tal fato denota a corretude do modelo. Em ambos os casos, os resultados foram satisfatórios e atenderam às expectativas dos projetistas e especialistas.

$\mathrm{Na}$ terceira fase de validação, para a construção do experimento, foram utilizados dados e taxas reais de um município do Rio Grande do Sul. Para tal, foram gerados três cenários a serem simulados no modelo: (a) cenário atual com variação atual das taxas; (b) cenário atual sem variação das taxas (estáticas); e (c) cenário futuro ideal, o qual é baseado em taxas da Holanda. Esse tipo de validação pode-se reconhecer como análise de sensibilidade, visto que foram utilizadas variáveis controladas nos inputs e verificadas as variações nos resultados. 0 detalhamento e quantificação de taxas para cada cenário simulado são apresentados a seguir na subseção 6.1.

\subsection{Cenários simulados no modelo}

Para a validação e experimentação do modelo de simulação desenvolvido foram gerados os seguintes cenários:

\subsubsection{Cenário atual com variação atual das taxas}

Para a simulação nesse cenário foram utilizados os dados e as taxas observadas no ano de 2010 (ASSOCIAÇÃO..., 2011), porém considerando a variação (crescimento ou diminuição) dessas ao longo dos anos. As taxas são apresentadas a seguir na Tabela 2 .

\subsubsection{Cenário atual sem variação de taxas (taxas estáticas)}

Para a simulação nesse cenário foram utilizados dados e taxas observados no ano de 2010 (ver Tabela 3) (ASSOCIAÇÃO..., 2011), mas considerou-se que as taxas permaneceriam estáticas em todo o tempo simulado.

\subsubsection{Cenário ideal futuro}

Para a simulação nesse cenário utilizaram-se dados e taxas observadas no ano de 2010 (ver Tabela 4), porém considerou-se que em 15 anos as taxas equiparar-se-ão às da Holanda em 2009 (EUROPEAN..., 2011), tendo em vista que elas são consideradas excelente parâmetro na área de gestão de RSU.

Tabela 1. Tipos de validação de sistemas.

\begin{tabular}{|c|c|}
\hline Tipo & Detalhes \\
\hline Validação nominal & $\begin{array}{l}\text { Baseada em especialistas, para verificar se o problema está bem formulado e contém as variáveis chave para a sua } \\
\text { representação. }\end{array}$ \\
\hline $\begin{array}{l}\text { Validação de } \\
\text { previsibilidade }\end{array}$ & Baseada em testes em laboratório do sistema, onde os resultados já são conhecidos e esperados. \\
\hline Rastreamento & $\begin{array}{l}\text { Impressão das variáveis de um programa logo após o evento ter ocorrido, para verificar se esse programa está } \\
\text { funcionando de acordo. }\end{array}$ \\
\hline Testes de Turing & $\begin{array}{l}\text { Adequados para sistemas de conhecimento. Testa a compatibilidade do resultado do sistema com aquele utilizado } \\
\text { por especialistas. }\end{array}$ \\
\hline Validação de subsistemas & Consiste na decomposição do sistema em módulos e sua validação em partes. \\
\hline Testes de campo & Consiste em fazer o sistema funcionar e verificar os erros de desempenho que ocorrem. \\
\hline Análise de sensibilidade & Consiste em fazer alterações controladas nos inputs do sistema e verificar as alterações nas saídas. \\
\hline Interação visual & Consiste em usar as capacidades visuais para observar o sistema em funcionamento. \\
\hline Avaliação do usuário & Consistem em consultar o usuário final do sistema e após o uso questionar sobre algumas caracteristicas do sistema. \\
\hline
\end{tabular}


Tabela 2. Taxas utilizadas no cenário simulado com a variação atual.

\begin{tabular}{|c|c|}
\hline Taxa & Valor \\
\hline Crescimento populacional & $\begin{array}{l}\text { Taxas atuais de natalidade e mortalidade - o crescimento populacional médio dos municípios do Rio Grande } \\
\text { do Sul foi de } 0,7 \% \text { (INSTITUTO..., 2010). }\end{array}$ \\
\hline Geração de resíduos & $\begin{array}{l}\text { A taxa média de geração de resíduos urbanos diária é de, aproximadamente, } 1,213 \mathrm{~kg} / \text { pessoa. A evolução da } \\
\text { geração anual é de } 2 \% \text {. }\end{array}$ \\
\hline \multirow{5}{*}{ Destino final dos resíduos } & $\begin{array}{l}\text { Aterro sanitário: Em 2010, aproximadamente 53\% dos resíduos gerados no Brasil tiveram como destino } \\
\text { aterros sanitários. A evolução anual da taxa de envio foi de aproximadamente } 1 \% \text {. Assume-se, para cálculo de } \\
\text { área ocupada, que em } 1 \mathrm{~m}^{3} \text { são depositados } 700 \mathrm{~kg} \text { de RSU compactado. }\end{array}$ \\
\hline & Reciclagem: A taxa de reciclagem média no Brasil é de 4,3\% e a evolução anual é de 3\%. \\
\hline & $\begin{array}{l}\text { Compostagem: A taxa média de compostagem no Brasil é de menos de 1\%. A evolução foi desconsiderada, } \\
\text { pois também é baixa. }\end{array}$ \\
\hline & Incineração: A taxa considerada foi de 0\%, pois esse tipo de disposição quase não é utilizado no Brasil. \\
\hline & $\begin{array}{l}\text { Tratamento inadequado: Refere-se aos resíduos sólidos dispostos de forma inadequada, os populares "lixões". } \\
\text { A taxa média no Brasil, em 2010, foi de } 42,4 \% \text { do total de resíduos. A redução desse tipo de destino final foi } \\
\text { de aproximadamente } 1 \% \text {. }\end{array}$ \\
\hline
\end{tabular}

Tabela 3. Taxas utilizadas no cenário simulado sem variação.

\begin{tabular}{|c|c|}
\hline Taxa & Valor \\
\hline Crescimento populacional & $\begin{array}{l}\text { Taxas atuais de natalidade e mortalidade - o crescimento populacional médio dos municípios do Rio Grande } \\
\text { do Sul foi de 0,7\% (INSTITUTO..., 2010), única taxa variável nesse cenário. }\end{array}$ \\
\hline Geração de resíduos & A taxa média de geração de resíduos urbanos diária é de, aproximadamente, 1,213kg/pessoa. \\
\hline \multirow{3}{*}{ Destino final dos resíduos } & $\begin{array}{l}\text { Aterro sanitário: Em 2010, aproximadamente 53\% dos resíduos gerados no Brasil foram destinados aos } \\
\text { aterros sanitários. Assume-se, para cálculo de área ocupada que em } 1 \mathrm{~m}^{3} \text { são depositados } 700 \mathrm{~kg} \text { de RSU } \\
\text { compactado. }\end{array}$ \\
\hline & $\begin{array}{l}\text { Reciclagem: A taxa de reciclagem média no Brasil é de } 4,3 \% \text {. } \\
\text { Compostagem: A taxa média de compostagem no Brasil é de menos de } 1 \% \text {. } \\
\text { lncineração: A taxa considerada foi de } 0 \% \text {, pois esse tipo de disposição quase não é utilizado no Brasil. }\end{array}$ \\
\hline & $\begin{array}{l}\text { Tratamento inadequado: A taxa média no Brasil, em } 2010 \text {, foi de } 42,4 \% \text { do total de resíduos, logo, nesse } \\
\text { cenário específico essa taxa manteve-se estática ao longo do tempo simulado. }\end{array}$ \\
\hline
\end{tabular}

\subsection{Experimento}

Após a definição dos cenários para a realização do experimento utilizando o modelo foram executadas as simulações. Como citado anteriormente, os dados utilizados nos três cenários foram de um município com, aproximadamente, 270 mil habitantes, do interior do Rio Grande do Sul, o qual possui aterro sanitário, aterro controlado (no modelo é considerado como tratamento inadequado), unidades de reciclagem e compostagem. 0 horizonte de tempo simulado no experimento foi de 30 anos, porém a configuração dessa variável fica a cargo do projetista.

Para a execução das simulações foi utilizado o simulador Vensim (VENTANA SYSTEMS, 2011) em uma estação de trabalho com processador Pentium Dual Core (G630) e $4 \mathrm{~Gb}$ de memória RAM. 0 tempo de execução da simulação dos três cenários foi da ordem de milionésimos de segundo. Os resultados obtidos no experimento realizado são apresentados na subseção 6.3 .

\subsection{Resultados obtidos}

Para o experimento realizado, alguns resultados, dentre os vários gerados, serão apresentados, tais como os relativos à geração de resíduos sólidos, área utilizada para aterro sanitário, quantidade de resíduo sólido enviada para reciclagem e para tratamento inadequado.

Primeiramente, quanto à geração de resíduos sólidos, verificou-se que o melhor cenário dentre os simulados é o cenário atual sem variação de taxas (taxas estáticas), pois nesse cenário mantém-se a taxa de geração atual e só ocorre o aumento na quantidade gerada de resíduos devido ao crescimento populacional, sendo que ao fim do tempo simulado (30 anos) a geração anual de RSU seria de, aproximadamente, 145 mil toneladas/ano. 0 cenário atual com variação atual de taxas e o cenário futuro ideal, em termos de geração de RSU, apresentam resultados menos eficientes, pois ao final do tempo simulado em ambos os casos a geração seria de, aproximadamente, 190 mil 
toneladas/ano. 0 cenário futuro ideal apresenta desempenho ruim nesse item, pois apesar do alto índice de tratamento dos RSU no país utilizado como parâmetro, a relação resíduo por habitante é maior que a taxa verificada no município analisado. Os resultados podem ser visualizados na Figura 5.

Com relação à área utilizada pelo aterro sanitário, ao longo dos 30 anos simulados (ver Figura 6), o cenário que apresentou o melhor resultado foi o cenário futuro ideal, pois a taxa de envio de RSU a partir do $15^{\circ}$ ano de simulação é da ordem de $1 \%$. A área estimada ao fim de 30 anos para o cenário futuro ideal foi de $816.000 \mathrm{~m}^{3}$, no cenário atual sem variação de taxas (taxas estáticas), a área estimada foi de $2.780 .000 \mathrm{~m}^{3}$ e no cenário atual com variação de taxas a área foi de $3.710 .000 \mathrm{~m}^{3}$. Os resultados gerados para essa variável específica, quando analisados periodicamente e em conjunção com a análise real de ocupação de aterros podem ser de grande importância para a determinação do tempo de ocupação total e para a execução de projetos para construção de novos aterros sanitários.

Em se tratando da quantidade enviada de RSU para a reciclagem, verificou-se após a simulação que o melhor resultado foi o cenário futuro ideal. Tal estimativa justifica-se pelo fato de o país utilizado como parâmetro para estabelecimento das taxas possuir índices elevados nesse tipo de tratamento dos resíduos. Outra constatação foi que, mantendo-se a evolução da reciclagem no município analisado, a quantidade de resíduos reciclados teria um crescimento de 350\% com o passar de 30 anos. Tal resultado apresenta a evolução nos índices de reciclagem de resíduos, porém ele tem pouca significância quando comparado à quantidade de resíduos reciclados utilizando-se os índices do cenário futuro ideal. Mantendo-se as taxas sem variação, a quantidade reciclada aumentaria em consonância com a quantidade de RSU gerada. Os resultados gerados para essa variável específica podem ser utilizados pelos gestores da área de RSU para o estabelecimento de programas de apoio à redução da geração de resíduos, programas de incentivo à segregação de resíduos na origem, bem como de incentivo à reciclagem e reutilização. Os resultados podem ser visualizados na Figura 7.

Com relação ao tratamento inadequado dos RSU, constata-se que dentre os três cenários simulados o pior caso é o do cenário atual sem variação de taxas, no qual, ao fim dos 30 anos simulados, a quantidade estimada de RSU inadequadamente tratada é da ordem de 65.700 toneladas/ano. A justificativa para tal resultado é que nesse cenário os índices de envio ao aterro sanitário e à reciclagem permanecem estáticos e existe o crescimento populacional.

Os crescentes aumentos da quantidade de RSU enviada aos aterros sanitários e da reciclagem de resíduos podem ser visualizados no cenário atual com variação de taxas onde, apesar da taxa de geração de resíduo por habitante e a população crescerem, a quantidade de RSU enviada para tratamento inadequado é menor do que no cenário sem variação de taxas. Ao final dos 30 anos simulados, a estimativa é

Tabela 4. Taxas utilizadas no cenário simulado com perspectivas futuras positivas.

\begin{tabular}{ll}
\hline \multicolumn{1}{c}{ Taxa } & \multicolumn{1}{c}{ Valor } \\
\hline Crescimento populacional & $\begin{array}{l}\text { Taxas atuais de natalidade e mortalidade - o crescimento populacional médio dos município do Rio Granc } \\
\text { do Sul foi de } 0,7 \% \text { (INSTITUTO..., 2010). }\end{array}$ \\
& $\begin{array}{l}\text { A taxa média diária considerada nesse cenário foi de, inicialmente, } 1,213 \mathrm{~kg} / \mathrm{pessoa,} \mathrm{considerando-se} \mathrm{a} \\
\text { sua evolução até alcançar a média de geração na Holanda, } 1,466 \mathrm{~kg} / \mathrm{pessoa,} \mathrm{no} 15^{\circ} \text { ano de simulação. } \\
\text { Geração de resíduos }\end{array}$ \\
& Posteriormente, essa taxa de crescimento manteve-se a mesma.
\end{tabular}

Aterro sanitário: Em 2009, aproximadamente 1\% dos resíduos gerados na Holanda tiveram por destino aterros sanitários. No início da simulação utilizou-se a taxa atual no Brasil (53\%), reduzindo-a até chegar à taxa holandesa no $15^{\circ}$ ano de simulação. Posteriormente manteve-se a mesma taxa até o fim do tempo simulado. Assume-se, para cálculo de área ocupada, que em $1 \mathrm{~m}^{3}$ são depositados $700 \mathrm{~kg}$ de RSU compactado.

Reciclagem: A taxa de reciclagem média no Brasil, que foi utilizada no início da simulação, é de 4\%. Evoluiuse a taxa, até o $15^{\circ}$ ano, quando ela alcança os $32 \%$ - taxa média de reciclagem na Holanda. Posteriormente, manteve-se a mesma taxa até o fim do tempo simulado.

Destino final dos resíduos Compostagem: A taxa média de compostagem no Brasil é de menos de 1\%. Evolui-se até os 28\%, taxa média da Holanda, no $15^{\circ}$ ano de simulação. Posteriormente, manteve-se a mesma taxa até o fim do tempo simulado.

Incineração: A taxa média de incineração da Holanda é de 39\%, no Brasil essa taxa é desprezível. Na simulação alcançou-se essa taxa no $15^{\circ}$ ano de simulação e, posteriormente, manteve-se a mesma taxa até o fim do tempo da simulação.

Tratamento inadequado: Na Holanda inexiste esse tipo de disposição. No modelo, a partir do $15^{\circ}$ ano de simulação, foi desprezado esse tipo de destino, pois os outros tipos de disposição final possuem capacidade para a quantidade de RSU gerada. 


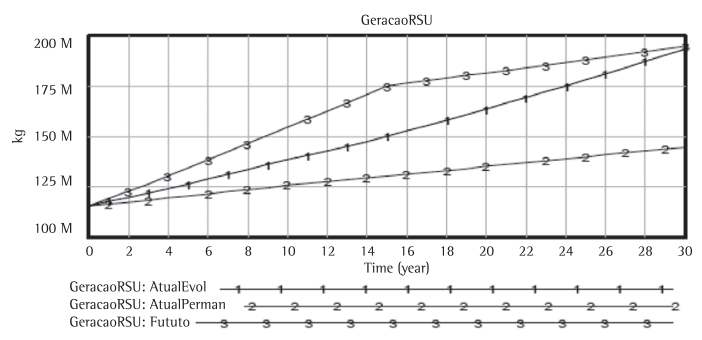

Figura 5. Resultados da variável referente à geração de resíduos sólidos urbanos.

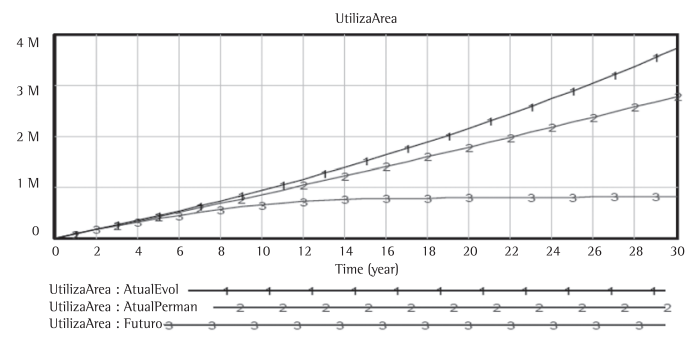

Figura 6. Resultados da variável referente à área (espaço) utilizada pelo aterro sanitário.

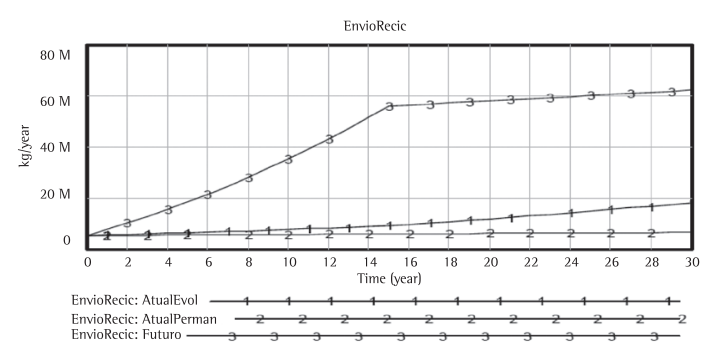

Figura 7. Resultados da variável referente à quantidade de resíduos enviada para reciclagem.

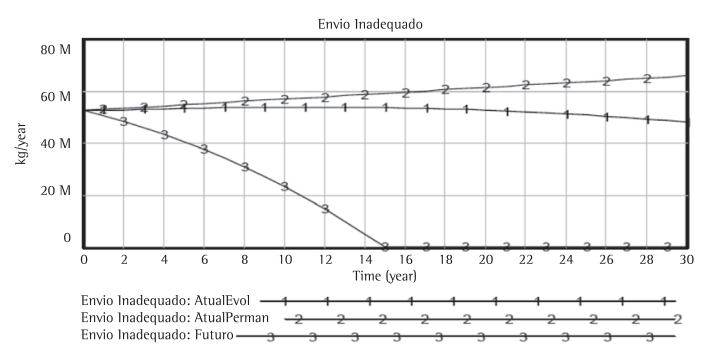

Figura 8. Resultados da variável referente à quantidade de resíduos enviada para tratamento inadequado.

de que 48 mil toneladas/ano de RSU terão tratamento inadequado.

No cenário futuro ideal existe uma redução significativa do envio de RSU para tratamento inadequado até $015^{\circ}$ ano simulado, pois nesse cenário concedeu-se ao município um prazo de 15 anos para adequar-se aos índices holandeses, ou seja, um valor aproximado de zero para esse tipo de destino final.
Os resultados simulados para essa variável podem ser visualizados na Figura 8.

\section{Considerações finais}

0 principal objetivo do trabalho foi apresentar o desenvolvimento, a validação e utilização de um modelo de simulação computacional para auxílio dos gestores da área de resíduos sólidos no processo decisório, no que se refere às estimativas sobre geração e possíveis destinos dos resíduos sólidos urbanos (RSU). Para o desenvolvimento do modelo foram utilizadas variáveis como crescimento populacional, quantidade média de resíduos gerada por habitante e taxas absorvidas por tipo de disposição final e, a partir dessas, o sistema foi capaz de estimar a quantidade de RSU a ser enviada para cada tipo de destino final, bem como a área total de aterro sanitário a ser ocupada pelos resíduos gerados pela população em um determinado período de tempo (que, no modelo desenvolvido, pode variar de horas a milhares de anos).

Através dos resultados gerados pela simulação, o gestor municipal poderá, por exemplo, estimar quando a capacidade de um aterro do seu município se esgotará e, antecipadamente, discutir, avaliar e decidir possíveis medidas necessárias para melhorar o aproveitamento do aterro atual como, por exemplo, aumentar a coleta seletiva e a compostagem, desenvolver campanhas para redução de RSU por parte da população e até mesmo iniciar o processo para a construção de um novo aterro sanitário. Optando por essa última alternativa, o modelo de simulação pode auxiliar no dimensionamento desse aterro, estimando até quando esse novo destino suportaria a demanda ou qual área seria necessária para depositar os resíduos sólidos gerados pela população do município ao longo de um número determinado de anos.

No artigo foram apresentados três cenários distintos, nos quais o modelo foi validado e avaliado utilizando-se dados de um município do Rio Grande do Sul. Os resultados gerados foram apresentados a especialistas, os quais utilizaram o modelo para testes, que satisfizeram-nos bem como confirmaram a hipótese dinâmica do modelo da pesquisa. Cabe a ressalva: os cenários foram gerados para esse experimento, o modelo de simulação pode ser configurado com o cenário que o usuário tiver necessidade, ou seja, é um modelo reconfigurável e aberto.

Com relação aos resultados gerados, para os cenários avaliados, o cenário denominado ideal futuro foi superior aos outros dois em quase todos os aspectos, porém na variável referente à estimativa de quantidade de resíduos gerada pela população, o 
cenário atual sem variação de taxas (taxas estáticas) foi melhor. Tal resultado deve-se ao fato de apesar de a Holanda possuir processos avançados de recuperação e reutilização de RSU, sua geração per capita de resíduo é bastante alta, tendo por base a do município simulado.

Como trabalho futuro pretende-se incluir novas variáveis no modelo como, por exemplo, população sazonal e novos tipos de destinação. Por fim, cabe ressaltar que os resultados obtidos nos experimentos satisfizeram as expectativas dos gestores municipais que o avaliaram.

\section{Referências}

ABELIOTIS, K. et al. Decision Support Systems in Solid Waste Management: A Case Study at the National and Local Level in Greece. Global NEST Journal, v. 11, n. 2, p. 117-126, 2009.

ASSOCIAÇÃO BRASILEIRA DE EMPRESAS DE LIMPEZA PÚBLICA E RESÍDUOS ESPECIAIS - ABRELPE. Panorama dos Resíduos Sólidos no Brasil-2010. ABRELPE, 2011.

BANI, M. S. et al. The Development of Decision Support System for Waste Management; a Review. World Academy of Science, Engineering and Technology, v. 49, p. 161-168, 2009.

BARLAZ, M. A. et al. Life-Cycle Study of Municipal Solid Waste Management - System Description. Washington: U.S. Environmental Protection Agency, 1995.

BHAT, V. N. A model for the optimal allocation of trucks for the solid waste management. Waste Management $\&$ Research, v. 14, p. 87-96, 1996.

BORENSTEIN, D.; BECKER, J. L. Validating Decision Support Systems. In: KENT, A.; WILLIAMS, J. G. (Eds.). Encyclopedia of Microcomputers. New York: Marcel Dekker, 2000.

BRASIL. Ministério das Cidades-Secretaria Nacional de Saneamento Ambiental. Sistema Nacional de Informações sobre Saneamento: diagnóstico do manejo de resíduos sólidos urbanos - 2008. Brasília: MCIDADESSNSA, 2010

CERIC, V. Simulation study of an automated guided-vehicle system in an Yugoslav hospital. Journal of the Operational Research Society, v. 41, n. 4, p. 299-310, 1990.

CHANG, N.; PARVATHINATHAN,G.; BREEDEN, J. B. Combining GIS with fuzzy multicriteria decision-making for landfill sitting in a fast growing urban region. Journal of Environmental Management, v. 87, p. 139-153, 2008. http://dx.doi.org/10.1016/j.jenvman.2007.01.011

CHANG, N.; WEl, Y. Siting recycling drop-off in urban area by genetic algorithm-based fuzzy multiobjective nonlinear integer programming modeling. Fuzzy Sets and Systems, v. 114, p. 133-149, 2000.

COSTI, P. et al. An environmentally sustainable decision model for urban solid waste management. Waste Management, v. 24, p. 277-295, 2004.

COVER, J. Introduction to System Dynamics. Powersim Press, 1996

DAELLENBACH, H. G.; McNICKLE, D. C. Decision making through systems thinking. Palgrave Macmillan, 2005.
DAVIES, M. N. Back-office process management in the financial services - a simulation approach using a model generator. Journal of the Operational Research Society, v. 45, n. 12, p. 1363-1373, 1994.

DEATON, M. L.; WINEBRAKE, J. J. Dynamic Modelling of Environmental Systems. Springer-Verlag, 2000.

DYSON, B.; CHANG, N. B. Forecasting municipal solid waste generation in a fast-growing urban region with system dynamics modeling. Waste Management, v. 25, n. 7, p. 669- 679, 2005. http://dx.doi.org/10.1016/j. wasman.2004.10.005

EUROPEAN COMMISSION STATISTICAL - EUROSTAT. Environment in the EU27 - Recycling accounted for a quarter of total municipal waste treated in 2009. Disponível em: <http://epp.eurostat.ec.europa.eu/cache/ ITY PUBLIC/8-08032011-AP/EN/8-08032011-AP-EN. PDF>. Acesso em: jun. 2011.

FINLAY, P. N. Introducing decision support systems. Oxford; Cambridge: Blackwell Publishers, 1994.

GHARAJEDAGHI, J. Systems thinking: managing chaos and complexity. 2nd ed. Elsevier, 2006.

HUANG, G. H.; BAETZ, B. W.; PATRY, G. G. Trash-Flow Allocation: Planning Under Uncertainty. Interfaces, v. 28, n. 6, p. 36-55, 1998. http://dx.doi.org/10.1287/inte.28.6.36

INSTITUTO BRASILEIRO DE GEOGRAFIA E ESTATÍSTICA - IBGE. Pesquisa Nacional de Saneamento Básico. IBGE, 2002. Disponível em: <http://www.ibge. gov.br/home/estatistica/populacao/condicaode vida/ pnsb/pnsb.pdf>. Acesso em: jun. 2011.

INSTITUTO BRASILEIRO DE GEOGRAFIA E ESTATÍsTICA - IBGE. Censo Demográfico 2010 IBGE, 2010. Disponível em: <http://www.ibge.gov.br/ home/download/estatistica.shtm>. Acesso em: jun. 2011.

KELTON, W. D.; SADOWSKI, R. P.; SADOWSKI, D. A Simulation with Arena. New York: McGraw-Hill, 1998.

KUM, V.; SHARP, A.; HARNPORNCHAI, N. A System Dynamics Study of Solid Waste Recovery Policies in Phnom Penh City. In: INTERNATIONAL CONFERENCE OF THE SYSTEM DYNAMICS SOCIETY, 23., 2005, Boston. Proceedings.. Boston: SDS, 2005.

LAW, A. M.; KELTON, W. D. Simulation Modeling \& Analysis. 2nd ed. McGraw-Hill, 1991.

MEADOWS, D. H.; MEADOWS, D. The limits to growth. New York: Universe Books, 1972.

MEADOWS, D. H.; RANDERS, J.; MEADOWS, D. Limits to Growth: The 30-Year Update. Chelsea Green, 2004.

MONTEIRO, J. H. P. et al. Manual de Gerenciamento Integrado de Resíduos Sólidos. Instituto Brasileiro de Administração Municipal - IBAM, 2001.

NANOHUB. NanoHUB.org - Online simulation and more for nanotechnology. Disponivel em: <http://nanohub.org>. Acesso em: abr. 2012.

NETTO, A. 0 Desperdício do Lixo. Jornal Zero Hora, p. 32, 09 set. 2001.

O'LEARY, P. R. et al. Decision Maker's Guide to Solid Waste Management. Washington: U.S. Environmental Protection Agency, 1999. v. 2.

PEGDEN, C. D.; SHANON, R. E.; SADOWSKI, R. P. Introduction to simulation using SIMAN. 2nd ed. McGraw-Hill, 1995.

PIDD, M. Simulating automated food plants. Journal of the Operational Research Society, v. 38, n. 8, p. 683-692, 1987. 
RESOL ENGENHARIA - RESIDDUOS SÓLIDOS. Trabalhos Técnicos. Disponível em: <http://www.resol.com.br/port/ trabTec_port_1.asp>. Acesso em: fev. 2008.

SALT, J. Tunnel Vision. OR/MS Today, v. 18, n. 1, p. $42-48,1991$.

SILVA, E. C. P. O impacto da gestão do tamanho da força policial na taxa de violência em Curitiba: Uma abordagem qualitativa sob o referencial da dinâmica de sistemas. 2006. Dissertação (Mestrado em Engenharia de Produção e Sistemas)-Pontifícia Universidade Católica do Paraná, Curitiba, 2006.

SIMON, H. A. Prediction and prescription in systems modeling. Operations Research, v. 38, p. 7-14, 1990. http://dx.doi. org/10.1287/opre.38.1.7

SIMONETTO, E. 0.; BORENSTEIN, D. A decision support system for the operational planning of solid waste collection. Waste Management, v. 27, p. 1286-1297, 2007. http:// dx.doi.org/10.1016/j.wasman.2006.06.012

STRAUS, L. M. Um modelo em dinâmica de sistemas para $o$ ensino superior. 2010. Dissertação (Mestrado em Administração)-Universidade Federal do Rio Grande do Sul, Porto Alegre, 2010.

SUFIAN, M. A.; BALA, B. K. Modeling of urban solid waste management system: The case of Dhaka city. Waste Management, v. 27, p. 858-868, 2007. http://dx.doi. org/10.1016/j.wasman.2006.04.011

TANSKANEN, J. H. Strategic planning of municipal solid waste management. Resources, Conservation and Recycling, v. 30, p. 111-133, 2000. http://dx.doi.org/10.1016/ S0921-3449(00)00056-2
TUNG, D. V.; PINNOI, A. Vehicle routing-scheduling for waste collection in Hanoi. European Journal of Operational Research, v. 125, p. 449-468, 2000. http://dx.doi. org/10.1016/S0377-2217(99)00408-7

UNITED NATIONS ENVIRONMENT PROGRAMME - UNEP. Solid Waste Management. CalRecovery Inc., 2005. v. 1.

VENTANA SYSTEMS. Vensim Simulation Software. Disponível em: <http://www.vensim.com>. Acesso em: mar. 2011.

WEINTRAUB, A.; MARTELL, D.; GUNN, E. Forest Management Challenges for Operational Researchers. European Journal of Operations Research, v. 104, p. 1-17, 1998. http://dx.doi.org/10.1016/S0377-2217(97)00329-9

WINZ, 1.; BRIERLEY, G. The Use of System Dynamics Simulation in Integrated Water Resources Management. In: International Conference of the System Dynamics Society, 25., 2007, Boston. Proceedings... Boston: SDS, 2007.

ZANIS, P. et al. Evaluation of near surface ozone in air quality simulations forced by a regional climate model over Europe for the period 1991-2000. Atmospheric Environment, v. 45, p. 6489-6500, 2011. http://dx.doi. org/10.1016/j.atmosenv.2011.09.001

ZUTSHI, A.; SOHAL, A. Environmental management systems auditing: auditors'experiences in Australia. International Journal Environment and Sustainable Development, v. 1, n. 1, p. 73-87, 2002. http://dx.doi.org/10.1504/ IJESD.2002.000719

\title{
Systems dynamics simulations for evaluating scenarios of urban solid waste generation and disposal
}

\begin{abstract}
This paper presents the development, validation and application of a simulation model using a systems dynamics methodology, which allows users and managers to evaluate and analyze scenarios in the context of urban solid waste generation and disposal. The developed model accounts for the following rates and parameters: rate of natural increase (birth rates and death rates), percentage of urban solid waste sent to each type of final disposal site and the amount of waste generated per capita. The simulated scenarios consider three distinct situations: (a) the current scenario with varying rates, (b) the current scenario with fixed rates and (c) a scenario with rates similar to those of the Netherlands. The model was validated through the analysis of future scenarios for a municipality in southern Brazil. Vensim- Ventana Systems was employed for the system modeling and development.
\end{abstract}

\section{Keywords}

Computational simulation. System Dynamics. Solid waste management. Decision-making process. Solid waste. 